\title{
Moraxella kingii sp.nov., a Haemolytic, Saccharolytic Species of the Genus Moraxella
}

\author{
By S. D. HENRIKSEN AND K. BØVRE \\ Kaptein W. Wilhelmsen og Frues Bakteriologiske Institutt, \\ University of Oslo, Rikshospitalet, Oslo, Norway \\ (Accepted for publication I8 October 1967)
}

SUMMARY

A new Moraxella species, Moraxella kingii, is described. It is characterized by $\beta$-haemolysis, acid production from glucose and maltose, and lack of catalase activity. The strain $4177 / 66$, isolated from a nose swab, is designated as the type strain.

\section{INTRODUCTION}

In 1960 the late Miss Elizabeth O. King of Atlanta, Georgia, U.S.A., sent a few strains to one of us with a request for an opinion as to whether these were strains of the genus Moraxella, and, if so, whether they belonged to any well-defined species. Two of the strains were $\beta$-haemolytic and saccharolytic. Morphologically and in growth characteristics they showed considerable likeness to Moraxella species, but the conclusion was that they represented a new species, and that Moraxella appeared to be the most suitable genus to allocate it to. Later we obtained Miss King's permission to include these strains in studies on the transformation reactions of Moraxella and related organisms which were then in progress, but it was our understanding that Miss King would describe this new species as soon as she found time to do so. Unfortunately this was prevented by her death. When we isolated an additional strain of this species from a nose culture in 1966, we felt that publication of a description of the new species was desirable. Inquiries to the Communicable Disease Center in Atlanta revealed that there was no objection to the inclusion of Miss King's strains in our study.

\section{METHODS}

Organisms. One of the strains (4177/66) was isolated in Oslo in 1966. Two other strains of the material had previously been received from the late Miss Elizabeth $O$. King, and 6 strains, also from her collection, were received from Dr R. E. Weaver (Atlanta). The strains were designated as follows (with source in parentheses): 294I (blood), 5530 (blood), 5963 (I) (blood), 8623 (blood), 957 I (throat), A I 702 (knee joint), A 247 I (bone lesion), A6042 (throat) and 4177/66 (nose).

Morphological, cultural and biochemical tests. With some modifications these were identical with the procedures previously described for the examination of Moraxella phenylpyrouvica (Bøvre \& Henriksen, $1967 b$ ). Haemolysis was studied on plates with ox, sheep, horse and rabbit blood in addition to the commonly used human blood agar medium. Tests for growth in citrate were made only in Koser's fluid medium. 
Examination for acid production from carbohydrates was extended to the use of ascites agar slants with $\mathrm{I} \%$ glucose, maltose, fructose, galactose, lactose, saccharose, arabinose, xylose, rhamnose, mannitol, dulcitol, sorbitol and glycerol, respectively.

$D N A$ base determinations. Guanine + cytosine $(\mathrm{G}+\mathrm{C})$ contents of DNA were determined by Dr W. Szybalski (Professor of Oncology, University of Wisconsin) with the $\mathrm{CsCl}$ buoyant density method on extracts prepared in the authors' laboratory.

Transformation methods. A quantitative procedure with short-term DNA exposure and a more sensitive, but less accurate approach, with continuous DNA exposure, were employed. The principles of mutant selection, transformation techniques, transformant assay and control measures, have been extensively described previously (Bøvre, 1964, I967a, c). The genetic marker used was exclusively one-step mutation to high streptomycin resistance.

\section{RESULTS}

\section{Morphological, cultural and biochemical characteristics}

Microscopical examination revealed coccoid to medium long diplo-bacteria with square ends, in pairs or chains. The bacteria were generally of rather small diameter as compared with previously studied Moraxella species. Pleomorphism was not pronounced in any of the strains. Microphotographs of two of the strains are given in Plate I, fig. I, 2. The organisms were Gram-negative, but in many instances some resistance to decolorization was observed. There were no distinct capsules. Motility was not observed.

All strains grew with small colonies, reaching diameters in the range $0.1-0.6 \mathrm{~mm}$. in culture on human blood agar plates after $20 \mathrm{hr}$ of incubation at $33^{\circ}$ and $37^{\circ}$. Single colonies of some strains increased to $\mathrm{I}-2 \mathrm{~mm}$. in size on prolonged incubation. The colonies were generally of the low hemispherical type. In some instances there was a tendency towards colony growth down into the medium, with resulting pitting of the surface. In these cases the colonies were flat, extending very little from the surface of the surrounding medium, and the growth mass appeared adherent to the medium. The colonies were non-pigmented, translucent or slightly opaque, with a smooth and sometimes mucoid appearance. Their consistency varied from soft, giving easy emulsification in physiological saline (strain 5530), to coherent, like gonococci, with resulting spontaneous agglutination (the other 8 strains). The latter phenomenon was only temporary, since complete emulsification occurred after a while in saline.

In all strains a distinct, but most often very narrow zone of $\beta$-haemolysis was observed around colonies on human blood agar. The haemolysis was more pronounced on plates with ox, sheep, horse or rabbit blood.

There was no anaerobic growth. In semifluid nutrient agar stab cultures growth could be observed down to about $5 \mathrm{~mm}$. below the surface. Very slight growth was observable with some of the strains at room temperature. There was no difference in growth capacity at $33^{\circ}$ and $37^{\circ}$. Most strains survived exposure to $45^{\circ}$ for 10 min., but not for $30 \mathrm{~min}$. None of the strains resisted heating to $49^{\circ}$ for Io min.

Growth in peptone media such as the indole and nitrate media used was absent or very scarce, as it was on Triple Sugar Iron Agar (Difco) and in the MR-VP medium. Only very little improvement of growth was observed when $10 \%(\mathrm{v} / \mathrm{v})$ horse serum was added to fluid peptone media or when heated blood was substituted for ordinary blood in agar plates. This phenomenon seems to distinguish our strains from other 
Moraxella kingii sp.nov.

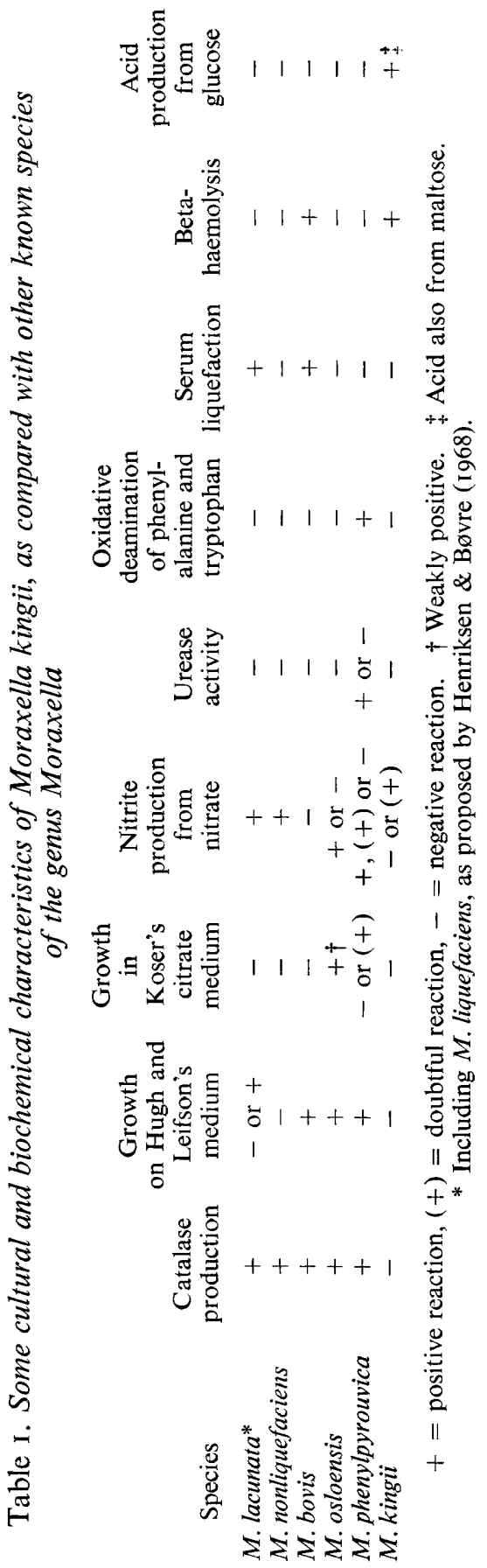


fastidious moraxellas, according to our experience. There was no satellite phenomenon with staphylococci. All strains were able to grow almost as well on high-quality nutrient agar as on the same medium with blood added.

The strains all had a remarkable inability to survive on blood agar plates at room temperature; cultures always became sterile in 6-12 days.

The catalase reaction was uniformly negative. This is in accordance with notes made by Miss Elizabeth $O$. King, placed at our disposal. The negative catalase reaction distinguishes our strains from all other known moraxellas, which have been extensively examined for this character in our laboratory (unpublished results). With the tetramethyl-p-phenylenediamine reagent all strains were strongly oxidase positive. With the dimethyl compound the reaction was also distinct with all strains, but perhaps weaker than with other known Moraxella species.

Strong acid production in I day was observed from glucose and maltose on ascites agar media. There was no apparent attack on the other carbohydrates and alcohols tested, except for very weak acid reactions in the galactose medium. No growth and no glucose attack was observed on Hugh and Leifson's medium. In I \% peptone liquid media with glucose growth was either absent or very weak, and only very late and irregular acid reactions were seen under these conditions.

No hydrogen sulphide or indole production was detected. Some results of cultural and biochemical tests are presented in Table $I$, together with a comparison with other moraxellas recently studied in this laboratory. The results of antibiotic sensitivity tests are listed in Table 2.

\section{DNA base composition}

The guanine + cytosine $(\mathrm{G}+\mathrm{C})$ content of all DNAs examined (from strains 294I, 5530, A I 702, 4I 77/66) was 44.5 moles $\%$.

Table 2. Sensitivity to antibiotics of the 9 strains of Moraxella kingii

\begin{tabular}{lcc}
\multicolumn{1}{c}{$\begin{array}{c}\text { Range } \\
\text { of inhibition } \\
\text { zones diam. } \\
(\mathrm{mm} .)\end{array}$} & $\begin{array}{c}\text { Approximate range } \\
\text { of minimum inhibitory } \\
\text { concentrations (m.i.c.)* }\end{array}$ \\
Penicillin & $37-54$ & $0.02-<0.005$ \\
Streptomycin & $27-34$ & $0.2-<0.01$ \\
Chloramphenicol & $36-45$ & $0.1-<0.03$ \\
Oxytetracycline & $25-30$ & $0.4-0.08$ \\
Eythromycin & $3 \mathrm{I}-45$ & $0.3-<0.008$
\end{tabular}

* Method of Ericsson, Høgman \& Wickman (I954). Minimal inhibitory concentration (m.i.c.) values calculated from zone diameters by means of regression equations for each antibiotic (Ericsson, 1960). M.i.c. given as i.u./ml. for penicillin, as $\mu \mathrm{g}$. $/ \mathrm{ml}$. for the other antibiotics.

\section{Transformation reactions}

The strain 4I77/66 was simultaneously exposed to marker DNAs of the same strain and of the strains 294I, 5530 and A I 702. There was a uniform distribution of interstrain transformation frequencies close to the frequency of intrastrain (autologous) transformation, as presented in Table 3. The resulting ratios of inter- to intra-strain transformation can be seen to range from $4 \cdot 6 \times \mathrm{IO}^{-1}$ to $9 \cdot 2 \times 10^{-1}$. Values of this order are consistent with a close taxonomic relationship and similar to those previously found between strains of one and the same species (see Bøvre, I967b). 
In Table 4 are presented results of five quantitative transformation experiments designed to elucidate the transformation compatibility of Moraxella kingii with other Moraxella species. No such compatibility was observed. Depending on the sensitivity of the individual experiments (i.e. the transformability or state of competence of the

Table 3. Quantitative streptomycin resistance transformation between strains of Moraxella kingii. Recipient strain 4177/66. Recipient count $7 \cdot 6 \times 10^{7} / \mathrm{ml}$.

Duration of DNA exposure $15 \mathrm{~min}$. Donor mutants selected at $500 \mu \mathrm{g}$. streptomycin $/ \mathrm{ml}$.; transformants selected at $50 \mu \mathrm{g} . / \mathrm{ml}$.

$\begin{array}{ccc}\text { Donor strain } & \begin{array}{c}\text { Transformants } \\ \text { per ml. }\end{array} & \begin{array}{c}\text { Ratio of } \\ \text { inter- to intrastrain } \\ \text { transformation }\end{array} \\ 2941 & 2.9 \times 10^{5} & 9.2 \times 10^{-1} \\ 5530 & 1.5 \times 10^{5} & 4.6 \times 10^{-1} \\ \text { A } 7702 & 2.2 \times 10^{5} & 6.9 \times 10^{-1} \\ 4177 / 66 & 3.2 \times 10^{5} & -\end{array}$

Table 4. Quantitative streptomycin resistance transformation in Moraxella, with Moraxella kingii as donor or recipient in interspecies experiments

Recipient strain

(recipient count $/ \mathrm{ml}$. in parentheses)

M. nonliq 7784
$\left(4.2 \times 10^{8}\right)$
M. osloensis 5873
$\left(9 \cdot 0 \times 10^{8}\right)$
M. bovis ATCC 10900
$\left(8 \cdot 0 \times 10^{7}\right)$
M. lacunata ATCC 11748
$\left(3.2 \times 10^{7}\right)$
M. kingii $4177 / 66$
$\left(6.0 \times 10^{7}\right)$

Donor strain

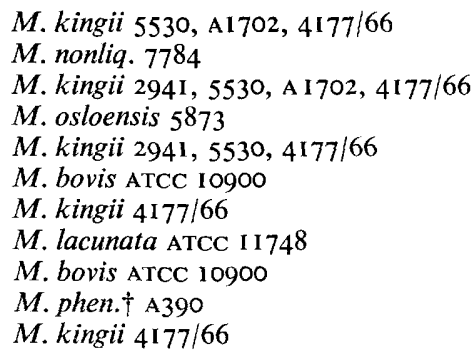

Ratio of inter-

Transformants to intrastrain per $\mathrm{ml}$. transformation

Duration of DNA exposure $15 \mathrm{~min}$. Donor mutants selected at $500 \mu \mathrm{g}$. of streptomycin per mi., transformants selected at $50 \mu \mathrm{g} . / \mathrm{ml}$.

* nonliq. = nonliquefaciens; + phen. = phenylpyrouvica.

recipients in question) ratios of inter- to intra-strain transformation between $M$. kingii and the other species were below $\mathrm{I}^{\cdot} 4 \times \mathrm{IO}^{-5}$ for $M$. liquefaciens, below $8.3 \times 10^{-6}$ for M. osloensis, below $2.3 \times 10^{-4}$ for $M$. bovis, below $3.7 \times 10^{-3}$ for $M$. lacunata and below $\mathrm{I} \cdot 0 \times 10^{-3}$ for $M$. phenylpyrouvica. In all the experiments of Table 4 , except for that with $M$. lacunata as recipient, the occurrence of transformants was also studied in continuous DNA exposure (without termination of DNA activity by DNase), which was performed in parallel. These experiments (not tabulated) were also negative, indicating that the transformation compatibility between $M$. kingii and the other species was considerably lower than the limits for these interactions listed above. Previous experiments with other organisms have shown that continuous DNA exposure was usually more than 100 times more sensitive for the detection of low transformation compatibility, as compared with the $15 \mathrm{~min}$. DNA exposure technique (Bøvre, I965).

In Table 5 are listed a few of the results obtained when highly competent Moraxella and Neisseria recipients were exposed continuously to DNAs of streptomycin- 
resistant Moraxella kingii on the one hand, and simultaneously to other distantly related donors on the other. It can be seen that when the conditions of transformation permitted the $M$. phenylpyrouvica 2863 donor to give 246 transformants in the $M$. osloensis 5873 recipient, several donors of $M$. kingii were still completely unable to elicit such transformants. Since the compatibility between these strains of $M$. phenylpyrouvica and $M$.osloensis is known to be of the order $6 \times \mathrm{IO}^{-6}$, as expressed by ratio of inter- to intra-strain transformation (Bøvre \& Henriksen, $1967 b$ ), ratios of transformation between $M$. kingii and $M$. osloensis would be lower than $3 \times 10^{-8}$. Whereas $M$. osloensis shows some very distant relations to all other moraxellas (Bøvre, I967a; Bøvre \& Henriksen, $1967 a, b$ ), there was no indication of any transformation compatibility between the former species and $M$. kingii.

Table 5. Semiquantitative comparison of low transformation frequencies (without termination of DNA exposure by DNase), with streptomycin resistance as the genetic marker

\begin{tabular}{|c|c|c|c|}
\hline Recipient strain & Donor strain & $\begin{array}{c}\text { Transformants } \\
\text { per plate }\end{array}$ & $\begin{array}{l}\text { Approximate } \\
\text { ratio of inter- to } \\
\text { intrastrain } \\
\text { transformation }\end{array}$ \\
\hline \multirow[t]{2}{*}{ Moraxella osloensis 5873} & M. phen. ${ }^{*} 2863$ & 246 & $6 \times 10^{-6} \ddagger$ \\
\hline & $\begin{array}{l}\text { M. kingii } 294 \mathrm{I}, 5530 \\
\text { А } 1702,4177 / 66\end{array}$ & 0 & $<3 \times 10^{-8}$ \\
\hline \multirow[t]{4}{*}{ Neisseria catarrhalis NE I I } & $M$. nonliq. +7784 & 3500 & $3 \times 10^{-5} \ddagger$ \\
\hline & M. osloensis 5873 & 276 & $2 \times 10^{-6} \ddagger$ \\
\hline & M. phen. 2863 & 613 & $5 \times 10^{-6} \ddagger$ \\
\hline & $M$. kingii $\mathrm{AI} 702$ & 0 & $<9 \times 10^{-9}$ \\
\hline \multirow[t]{3}{*}{ Neisseria ovis $199 / 55$} & M. osloensis 5873 & 1300 & $3 \times 10^{-5}$ \\
\hline & M. phen. 2863 & $56 \mathrm{I}$ & $1 \times 10^{-5}$ \\
\hline & $M$. kingii $\mathrm{A} 1702$ & 0 & $<3 \times 10^{-8}$ \\
\hline
\end{tabular}

Donor mutants selected at $500 \mu \mathrm{g}$. of streptomycin per ml., transformants selected at $50 \mu \mathrm{g}$. per $\mathrm{ml}$. For methodology, see Bøvre (1967a).

$*$ phen. $=$ phenylpyrouvica $; \dagger$ nonliq. $=$ nonliquefaciens .

$\ddagger$ Previously estimated (Bøvre, 1967a; Bøvre \& Henriksen, 1967b).

Similar comparisons of low transformation frequencies in continuous DNA exposure were performed with Neisseria catarrhalis and $N$. ovis recipients (Table 5). These experiments revealed no transformants with Moraxella kingii donors under conditions where $M$. nonliquefaciens, $M$. osloensis and $M$. phenylpyrouvica donors elicited hundreds or thousands of transformants in the same recipients. The activities of the latter three donors had been estimated previously (Bøvre, 1967a; Bøvre \& Henriksen, $1967 b$ ), and by means of these values the ratio of inter- to intra-strain transformation between $M$. kingii and $N$. catarrhalis would be situated below $9 \times 10^{-9}$, and between $M$. kingii and $N$. ovis the ratio would be below $3 \times 10^{-8}$. So far there is no indication of any transformation compatibility between $M$. kingii and $N$. catarrhalis or N. ovis, in contrast to the distinct, but often very distant relations in these terms previously found between all other moraxellas and these two Neisseria species.

Continuous DNA exposure experiments were also performed to detect compatibility between Moraxella kingii and Neisseria flavescens or $N$. meningitidis, with negative results. However, the recipients of these experiments were not of particularly high competence, so that a final decision cannot be made as regards absence of affinity between $M$. kingii and the latter Neisseria species. 


\section{DISCUSSION}

The results indicate that the strains included in this study are a homogeneous group which is sufficiently distinct from all bacterial species known to us, to deserve the status of a species. This new species shares many of the characteristics of the genus Moraxella in morphology, cultural behaviour, oxidase reaction and antibiotic sensitivity, and it has a DNA base composition close to the range of the other Moraxella species. Like the majority of the latter it is transformable. On the other hand, the group shows marked differences from all the other known Moraxella species, the most conspicuous of which are the ability to produce acid from glucose and maltose, and the lack of catalase.

Flamm (1956) described a new species, Moraxella saccharolytica, which, like our organism, produced acid from glucose, maltose, fructose, saccharose, glycerol, starch; but not from arabinose, dulcitol, galactose, inositol, lactose, mannitol, melizitose, raffinose, rhamnose, salicin, sorbitol, xylose. It was non-haemolytic and it further differed from our strains in the following characters: good growth at $22^{\circ}$, viable on blood agar for 4 weeks, gelatin liquefaction, catalase production, and resistance to streptomycin, chloramphenicol and tetracyclines. As far as we know strains of this organism have not been maintained, at least no strain has been available to us; but it seems quite obvious that $M$. saccharolytica differs from our strains in too many characters to belong to the same species. The study of new isolates of $M$. saccharolytica would be necessary to determine the taxonomic position of this species and its relationships to other Moraxella species.

Whereas other Moraxella species show some, even if very slight, signs of mutual compatibility in transformation experiments, no sign of compatibility of the new species with other Moraxella species or with Neisseria could be detected. This suggests that the new species is more distantly related to the other Moraxella species than the latter are to one another. Nevertheless, we feel that the likeness to other species of the genus Moraxella is sufficient to admit the new species to this genus; we find at present no reasonable alternative.

The habitat of the new species is uncertain in view of the fact that so few strains are known; 3 of the 9 strains were isolated from throat or nose of patients. It may be pointed out that this organism could very easily be mistaken for $\alpha$ - or $\beta$-haemolytic streptococci or for haemolytic Haemophilus species, and it is possible that a systematic search for this organism might reveal that it is less rare than it may appear.

We name this species Moraxella kingii in honour of the late Miss Elizabeth O. King, who did the pioneer work on this species and who collected most of the existing strains. The strain $4177 / 66$ is designated as type strain. It is being deposited in the National Collection of Type Cultures (Great Britain) and in the American Type Culture Collection. A condensed description of the species follows.

\section{Description of Moraxella kingii sp.nov.}

Micromorphology. Organisms coccoid to medium-sized rods, somewhat smaller than those of other Moraxella species, with square ends, in pairs and chains. Gramnegative, with some tendency to resist decolorization. Not capsulated. Non-motile. No endospores.

Colonies. Delicate, translucent or slightly opaque, $0 . \mathrm{I}-0.6 \mathrm{~mm}$. in diameter after 
$20 \mathrm{hr}$ on blood agar. Usually low hemispherical, smooth, but occasionally flat with some pitting of the agar surface, or of a mucoid appearance. No pigment. Consistency soft or coherent, in the latter case showing transient spontaneous agglutination in saline. Colonies surrounded by distinct zones of beta-haemolysis.

Relation to oxygen. Aerobe.

Temperature. Slight growth at room temperature (about $20^{\circ}$ ). Optimal growth at $33-37^{\circ}$. Usually killed by exposure to $45^{\circ}$ for Io-30 min.

Growth requirements. Comparatively fastidious. Little or no growth in peptone media. No growth on Hugh \& Leifson's medium. Practically no improvement of growth by addition of $10 \%(\mathrm{v} / \mathrm{v})$ horse serum to liquid peptone media. Growth on high quality nutrient agar almost as good as on blood agar. No apparent need for $\mathrm{X}$ or V factors.

Viability. Blood agar cultures become sterile within 6-1 2 days at room temperature.

Biochemical reactions. Catalase reaction negative. Oxidase reaction strongly positive with tetramethyl-, less strongly positive with dimethyl-p-phenylenediamine. Nitrate not reduced or slight reduction. Acid rapidly produced on ascites fluid agar slopes from glucose and maltose. Trace of acid from galactose. No acid from fructose, lactose, saccharose, arabinose, xylose, rhamnose, mannitol, dulcitol, sorbitol, glycerol. Gelatin or serum not liquefied. Indole or hydrogen sulphide not produced. Urease negative. No oxidative deamination of phenylalanine or tryptophan.

Antibiotic sensitivity. Highly sensitive to penicillin, streptomycin, chloramphenicol, oxytetracycline, erythromycin.

Habitat. Strains have been isolated from throat, nose, blood, bone lesion and joint.

Pathogenicity. Uncertain, probably low.

DNA base composition. 44.5 mole \% guanine+cytosine $(\mathrm{CsCl}$ buoyant density method).

Genetic compatibilities. Strains are mutually compatible with ratios of interstrain to intrastrain transformant numbers close to I. No compatibility detected with other Moraxella or with Neisseria species.

Our thanks are due to Drs W. B. Cherry and R. E. Weaver, both of Atlanta, Georgia, U.S.A., for allowing us to use Miss King's strains in the study, and for kindly sending us additional strains from her collection. We are most grateful to Professor W. Szybalski for performing the DNA base determinations.

\section{REFERENCES}

BøVRE, K. (I964). Studies on transformation in Moraxella and organisms assumed to be related to Moraxella. I. A method for quantitative transformation in Moraxella and Neisseria, with streptomycin resistance as the genetic marker. Acta path. microbiol. scand. 6r, 457.

Bøvre, K. (1965). Studies on transformation in Moraxella and organisms assumed to be related to Moraxella. 4. Streptomycin resistance transformation between asaccharolytic Neisseria strains. Acta path. microbiol. scand. 64, 229.

Bøvre, K. (1967a). Studies on transformation in Moraxella and organisms assumed to be related to Moraxella. 7. Affinities between oxidase positive rods and neisseriae, as compared with group interactions on both sides. Acta path. microbiol. scand. 69, 92.

BøvRE, K. (1967b). Transformation and DNA base composition in taxonomy, with special reference to recent studies in Moraxella and Neisseria. Acta path. microbiol. scand. 69, I 23. 


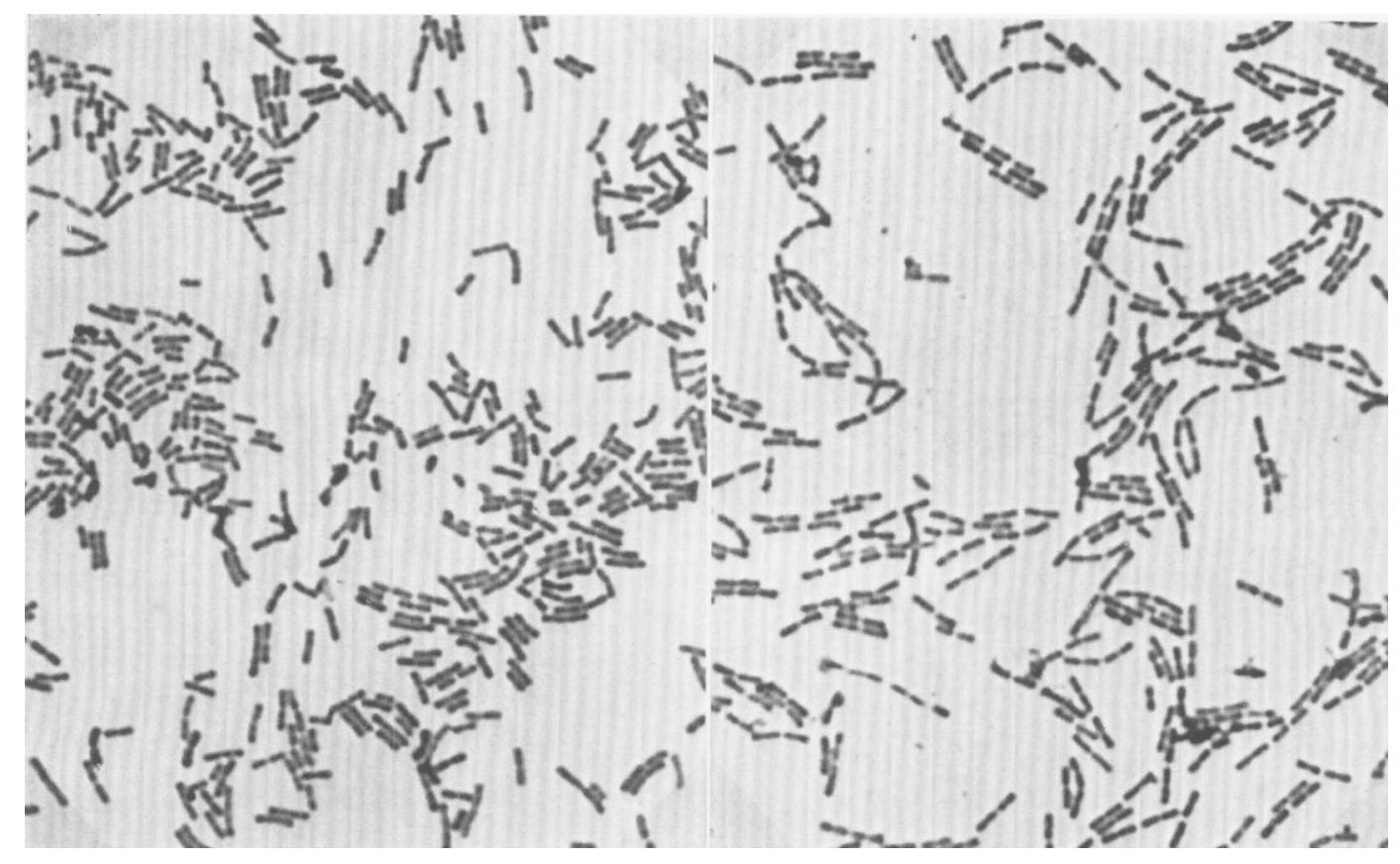

Fig. I

Fig. 2 
Bøvre, K. (1967c). Moraxella and Neisseria. Transformation, DNA analysis and taxonomy. Dr. med. Thesis, Faculty of Medicine, University of Oslo. Oslo: Universitetsforlaget.

Bøvre, K. \& Henriksen, S. D. (I967a). A new Moraxella species, Moraxella osloensis, and a revised description of Moraxella nonliquefaciens. Int. J. system. Bact. 17, 127.

Bøvre, K. \& Henriksen, S. D. (1967b). A revised description of Moraxella polymorpha Flamm I957, with a proposal of a new name, Moraxella phenylpyrouvica for this species. Int. J. system. Bact. (in the Press).

Ericsson, H. (1960). Rational use of antibiotics in hospitals. Studies on laboratory methods and discussion of the biological basis for their clinical application. Scand. J. clin. Lab. Invest. 12, Suppl. 50.

Ericsson, H., Høgman, C. \& Wickman, K. (1954). A paper disc method for determination of bacterial sensitivity to chemotherapeutic and antibiotic agents. Scand. J. clin. Lab. Invest. 6, Suppl. I I.

Flamm, H. (1956). Moraxella saccharolytica (sp.n.) aus dem Liquor eines Kindes mit Meningitis. Zentbl. Bakt. (I. Abt. Orig.) 166, 498.

Henriksen, S. D. \& Bøvre, K. (1968). The taxonomy of the genera Moraxella and Neisseria. J. gen. Microbiol. 5r, 387.

\section{EXPLANATION OF PLATE}

Fig. I. Moraxella kingii, strain 4177/66 (type strain). Stained by Gram's method. × I600.

Fig. 2. Moraxella kingii, strain A I702. Stained by Gram's method. $\times 1600$. 